\title{
Meso to Atomic Scale Microstructural Changes During Ageing of NCM Li-ion Battery Materials
}

\author{
Jacob R. Jokisaari ${ }^{*}$, Jinglong Guo ${ }^{1}$, Javier Bareno ${ }^{2}$, Daniel Abraham² and Robert F. Klie ${ }^{1}$ \\ 1. University of Illinois at Chicago, Department of Physics, Chicago, IL, USA. \\ 2. Argonne National Laboratory, Lemont, IL, USA. \\ * Corresponding author: joki@umich.edu
}

Layered oxide materials are commonly utilized in lithium-ion battery cells. Examples include $\mathrm{LiCoO}_{2}$, and compounds of composition $\mathrm{Li}\left(\mathrm{Mn}_{\mathrm{x}} \mathrm{N}_{\mathrm{y}} \mathrm{Co}_{1-\mathrm{x}-\mathrm{y}}\right) \mathrm{O}_{2}$, the so called NMCs. These oxides, are suitable for high-voltage operation, and could increase the energy density of Li-ion batteries. Mechanisms of loss of capacity, rise of impedance, capacity loss, and other related metrics are of importance for these next generation batteries. The structural modifications involved in these processes are not entirely understood, particularly processes occurring at the atomic scale, due to the difficulty in imaging them directly. The first step in imaging is sample preparation, and traditional powder sample methods pose problems for these materials. The particle size in the majority of battery materials is too large for direct analysis by TEM. Powder preparation methods necessarily result in a loss of information due to homogenization of the many components of the electrode and loss of all associations between the fragments that become thin enough for atomic-resolution imaging.

To avoid these difficulties, a focused Ga-ion beam can be employed to extract a section from the cathode. This has many benefits, preserving the shape of the secondary particle, resulting in a specimen of relatively uniform thickness which preserves the geometric relationship between the primary crystallites, revealing structural changes, grain boundary changes, and comparison between the surface and bulk of both the primary particles, and of the larger agglomerate. Sections were extracted from both aged and pristine electrodes prepared as battery electrodes under the same conditions. Images showing the FIB sectioning process and low magnification low-angle annular dark field images of pristine and aged specimens are shown in Figure 1. Even at low magnification, there are obvious changes. It has previously been noted that there is a considerable volume change with intercalation/deintercalation in NMC materials,[1] which here can be seen to result in increasing separation of the primary particles at the meso scale. This separation directly impacts the electrochemical performance, as the particles lose electrical contact with each other. EDS mapping between particles in Fig. 1e) shows the presence of fluorine and phosphorus, indicating penetration of electrolyte between the crystallites.

Figure 2 shows LAADF images from several different regions around a representative particle. Examination at atomic resolution reveals several details. One is the initiation points of the surface corrosion, where a spherical loss of density can be seen, likely at a linear defect. This is particularly obvious in the LAADF images, where the local distortion appears bright due to diffraction contrast. As corrosion advances, linear steps features appear. The particular extent of corrosion may depend on the misfit between the two crystals, as different behavior can be seen at different interparticle interfaces.

Close examination of the primary particle edges (Fig. 2e-e), shows atomic-scale alterations in structure. The particle presents a layered structure viewed on a $<010>$ axis. However, near the edges of the particles up to $\sim 10-20 \mathrm{~nm}$ into the interior, there is the appearance of higher contrast in the interlayer regions, with alternating high/low contrast along the planes at $\sim 30 \mathrm{deg}$ angles. This may be due to the 
formation of local, spinel-like domains on a $<110>$ in the crystal. These increase the impedance to ion transfer, and contribute to a decrease in the electrochemical performance. At the very surface of the particle, 1-2 nm in, there is the appearance of a 'rock-salt' like phase. Surface reconstructions resulting in similar contrast were seen both in pristine and aged specimens, and appears to be common in these materials. The extent appears larger in the aged material, and was associated with the more subtle crystal changes above which extend far into the primary crystallites.

The combination of the subtle atomic-scale crystal transformation and the meso-scale disassociation of the primary crystallites in the electrode particles, along with additional features, all show how relatively subtle nanoscale structural changes contribute to ageing phenomena in NCM battery materials.[2]

\section{References:}

[1] F Schippera et al., J. of The Electrochem. Soc. 164 (2017), p. A6220.

[2] This work was funded in part by a grant from Argonne Joint Center for Energy Storage Research (JCESR).
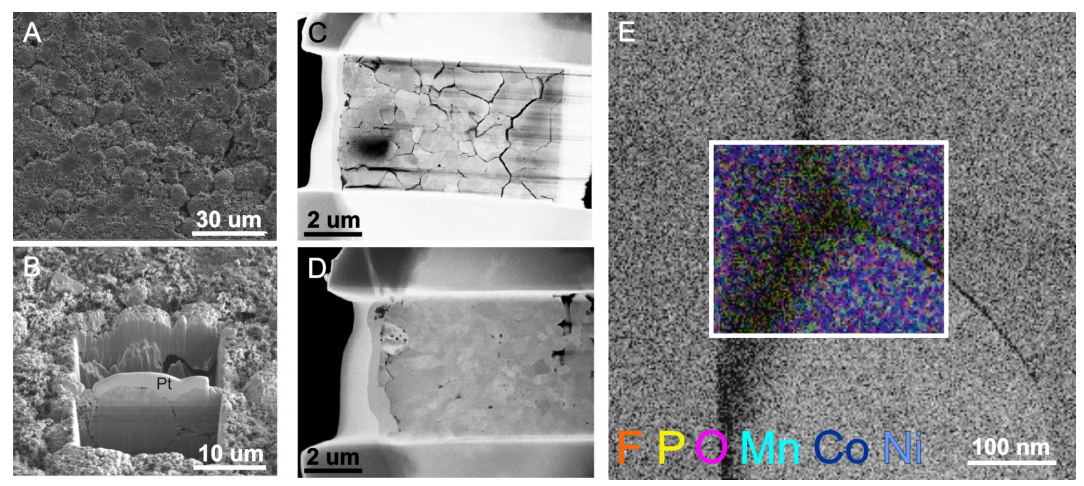

Figure 1. A) SEM image of electrode surface B) FIB cut trench of liftout sample C) FIB cross section of cycled sample D) FIB cross section of pristine sample D) EDS map showing elements from electrolyte at internal grain boundaries.
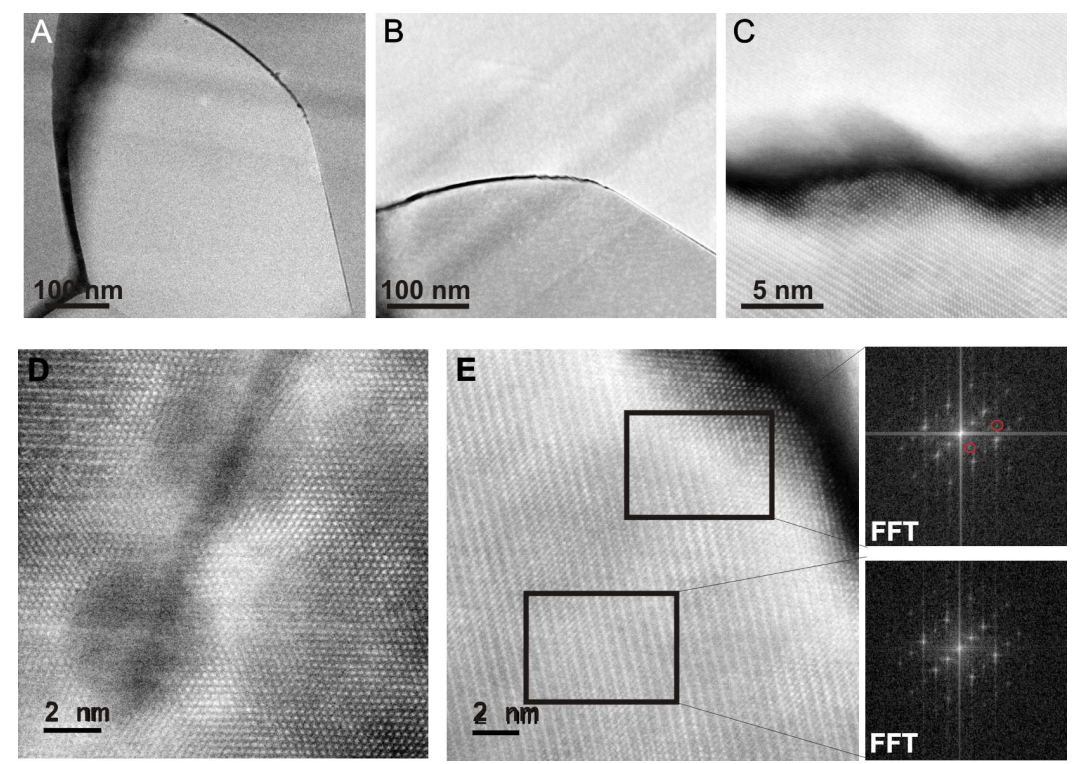

Figure 2. A) LAADF image of an internal particle B) edge of the same particle showing steps C) high magnification LAADF image of steps D) high resolution image of interface showing spherical corroded areas E) FFT analysis of edge of crystal shows extra spots $\sim 10 \mathrm{~nm}$ into the surface of the particle. 